\title{
Neumonía por coinfección de Aspergillus fumigatus y Pneumocystis jirovecii en un paciente no VIH: Reporte de caso
}

\author{
Pneumonia due to Aspergillus fumigatus and \\ Pneumocystis jirovecii coinfection in a non-HIV- \\ infected patient: Case report.
}

Correspondencia

Martín Humberto Iglesias Guzmán mig0013@hotmail.com

Recibido: 08/08/2021

Arbitrado por pares

Aprobado: 29/11/2021

Citar como: Martínez Montalvo CM, Perez MA, Fuentes-Lacouture MC, Bravo-Mena K, Leal-Bernal SF, Velasco YM, Charria Caicedo $M$, Estrada Serrano N. Neumonía por coinfección de Aspergillus fumigatus y Pneumocystis jirovecii en un paciente no $\mathrm{VIH}$ : Reporte de caso. Acta Med Peru. 2021;38(4):

313-8. doi: https://doi.org/10.35663/ amp. 2021.384 .2174
Carlos Mauricio Martínez Montalvo ${ }^{1, a}$, Manuel Antonio Perez ${ }^{1, a}$, María Cynthia Fuentes-Lacouture ${ }^{1, a}$, Karime Bravo Mena ${ }^{2, b}$, Sofía Fernanda Leal Bernal 3,b , Yulieth Magdalena Velasco 4,b, Melissa Charria Caicedo 5,b, Natalia Estrada Serrano 5,b.

Universidad del Rosario, Bogotá, Colombia.

Universidad Santiago de Cali, Colombia.

Universidad de Nariño, Pasto, Colombia.

Universidad del Cauca, Popayán, Colombia.

Fundación Universitaria San Martin, Cali, Colombia.

Residente de Medicina Interna

Médico general

\section{RESUMEN}

La aspergilosis pulmonar invasiva es una enfermedad presente principalmente en pacientes inmunocomprometidos con alta carga de mortalidad. La neumonía por Pneumocystis jirovecii es una infección oportunista potencialmente mortal que afecta a pacientes inmunocomprometidos por diversas etiologías. La coinfección por estos patógenos en pacientes inmunocompetentes es inusual. Reportamos un caso de un paciente sin las causas tradicionales de inmunocompromiso en el desarrollo de una neumonía en coinfección por Aspergillus fumigatus y Pneumocystis jirovecii.

Palabras clave: Aspergilosis pulmonar invasiva; Pneumocystis jirovecii; Neumonía por Pneumocystis; Cirrosis hepática; Colombia.

\begin{abstract}
Invasive pulmonary aspergillosis is a condition that mainly occurs in immunosuppressed patients, and it has a high mortality rate. Pneumonia caused by Pneumocystis jirovecii is a potentially lethal opportunistic infection affecting immunosuppressed patients with different etiology. Coinfection by Aspergillus and $P$. jirovecii in immunocompetent patients is unusual. We report a case of a patient with no common causes of immunosuppression who developed pneumonia coinfection caused by Aspergillus fumigatus and Pneumocystis jirovecii.
\end{abstract}

Keywords: Invasive Pulmonary Aspergillosis; Pneumocystis jirovecii; Pneumocystis pneumonia; Liver Cirrhosis; Colombia. 


\section{INTRODUCCIÓN}

La neumonía por Pneumocystis jirovecii es una infección oportunista potencialmente mortal que afecta principalmente a pacientes inmunocomprometidos por diversas etiologías incluyendo VIH, neoplasias, quimioterapia, uso de glucocorticoides o receptores de trasplante ${ }^{[1]}$, con diferentes manifestaciones clínicas descritas dependiendo de la causa del inmunocompromiso ${ }^{[2]}$. La aspergilosis pulmonar invasiva es una infección que afecta predominantemente a pacientes inmunocomprometidos, en especial a pacientes con antecedente de trasplante, neutropénicos o con altas dosis de glucocorticoides ${ }^{[3,4]}$.

Si bien se han descrito casos de estas infecciones en pacientes inmunocompetentes ${ }^{[5,6]}$ en su mayoría la presentación es en pacientes con algún grado de inmunocompromiso. Debido a lo anterior se presenta un caso en paciente no VIH de coinfeccion entre dos gérmenes oportunistas con desenlace fatal.

\section{Descripción del caso}

Paciente masculino de 42 años, con antecedentes de cirrosis hepática Child B por síndrome de sobreposición (hepatitis autoinmune y cirrosis biliar primaria) hace 3 años sin manejo, con múltiples complicaciones derivadas de la misma dadas principalmente por encefalopatía hepática a repetición y várices esofágicas, además con epilepsia e hipertensión arterial. Se encontraba hospitalizado por encefalopatía West Haven grado II, y donde había recibido manejo antibiótico con piperacilina/ tazobactam por 24 horas, sin un foco infeccioso claro. Ingresa referido a nuestra institución para valoración por hepatología y continuidad de estudios pre- trasplante.

Al examen físico se observaba ictericia generalizada y ascitis grado 2 con signos vitales dentro de límites normales. En paraclínicos de ingreso se observaba con leucocitosis asociado a neutrofilia, anemia de volúmenes normales, trombocitopenia y un perfil de función hepática con elevación de transaminasas, un Factor R de 1.9 (Patrón colestásico); Se amplían estudios de etiología

Tabla 1. Reporte de Exámenes de Laboratorio durante la hospitalización del paciente.

\begin{tabular}{|c|c|c|c|}
\hline \multicolumn{2}{|c|}{ Hemograma } & \multicolumn{2}{|c|}{ Tamizaje Infeccioso } \\
\hline Leucocitos (/ul) & 15000 & AgsHB & Negativo \\
\hline Neutrófilos (\%) & 90 & DNA VHB & Negativo \\
\hline Linfocitos (\%) & 5 & $\mathrm{AC} \mathrm{VHC}$ & Negativo \\
\hline Hemoglobina (g/dl) & 10 & RPR & Negativo \\
\hline Plaquetas(/ul) & 103,000 & $\mathrm{VIH}$ & Negativo \\
\hline PCR (mg/dL) & 250 & Carga viral CMV & Negativo \\
\hline VSG (mm/h) & 100 & Urocultivo - Hemocultivos & Negativo \\
\hline \multicolumn{2}{|c|}{ Química Sanguínea } & Cultivo de L. Peritoneal & Negativo \\
\hline Bilirrubina(mg/dL) & 26.8 & Pro-calcitonina & $<0.1 \mathrm{ng} / \mathrm{ML}$ \\
\hline Bilirrubina directa & 19.2 & Gota gruesa seriada & Negativo \\
\hline AST (IU/L) & 126 & Antígenos febriles & Negativo \\
\hline ALT (IU/L) & 202 & & \\
\hline $\mathrm{FA}(\mathrm{IU} / \mathrm{L})$ & 389 & \multicolumn{2}{|c|}{ Gases arteriales } \\
\hline GGT (IU/L) & 315 & $\mathrm{PH}$ & 7.32 \\
\hline PT (sec)/INR & $18,3 / 1.3$ & PCO2 & 45 \\
\hline Creatinina(mg/dL) & 0.9 & PO2 & 62 \\
\hline BUN (mg/dL) & 18.2 & $\mathrm{HCO} 3$ & 22 \\
\hline Albumina (g/L) & 2.5 & $\mathrm{BE}$ & -3 \\
\hline Potasio (Meq/L) & 4.19 & PAFI & 193 \\
\hline Cloro (Meq/L & 98.9 & Lactato & 2.5 \\
\hline Sodio (Meq/L) & 135 & & \\
\hline
\end{tabular}

PCR (Proteína C reactiva), VSG (Volumen de sedimentación globular), AST (Aspartato amino transferasa), ALT (Alanino amino transferasa), FA (Fosfatasa alcalina), GGT (Gama glutil transferasa), PT (Tiempo protrombina), BUN (Nitrógeno ureico), AGsHB (Antígeno superficie de hepatitis B), DNA VHB (Carga viral virus hepatitis B) , VIH ( Virus inmunodeficiencia humana), CMV (Citomegalovirus), PCO2 (Presión dióxido carbono), PO2 (Presión oxigeno), HCO3 (Bicarbonato), BE (Base exceso), PAFI (Presión oxigeno/ Fracción inspirado de oxigeno). 
infecciosa con virus hepatotropos negativo, estudio liquido peritoneal negativo para peritonitis bacteriana espontánea, uroanálisis sugestivo de infección (Ver Tabla 1) y radiografía de tórax dentro de límites normales (Ver Figura 1). Debido a lo anterior se inició manejo para encefalopatía con rifaximina y lactulosa, con indicación de manejo adicional de ácido ursodesoxicolico y cubrimiento antibiótico dando continuidad con piperacilina/Tazobactam dosis de 4.5 gramos intravenoso cada 6 horas. Ante deterioro clínico de paciente con aumento de encefalopatía y persistencia de fiebre, alteración síntesis hepática (INR 2,5), progresión de anemia y trombocitopenia, se realiza toma de hemocultivos y estudio de líquido cefalorraquídeo con líquido dentro de límites normales. Se amplían estudios por nexo de procedencia del paciente de zona endémica selvática con gota gruesa seriada y antígenos febriles los cuales son negativos; se inicia cubrimiento Ceftriaxona 2 gramos/día intravenoso y Doxiciclina 100 mg vía oral cada 12 horas en día 2 de hospitalización.

A las 36 horas se evidencia requerimiento de oxigeno con Fio2 $32 \%$ con reporte de gases arteriales con trastorno moderado de oxigenación ( $\mathrm{PaFI} 193$ ) con acidosis respiratoria compensada con lactato 2,5 sin signos de dificultad respiratoria y en el momento con mejoría de encefalopatía; Se decide toma tomografía de tórax con hallazgos de opacidades en vidrio esmerilado multilobares de predominio periférico y un nódulo cavitado en el lóbulo inferior izquierdo (Figura 2). Fue llevado a fibrobroncoscopia y lavado bronco alveolar con resultado en estudio citológico con leucocitos aumentados, tinciones negativas y galactomanano negativo (Tabla 2).

Ante sospecha infecciosa en nódulo cavitado se difirió el uso de esteroides de patología de base, adicionalmente se inicia cubrimiento con anfotericina B liposomal $0.5 \mathrm{mg} / \mathrm{kg} / \mathrm{dia}$

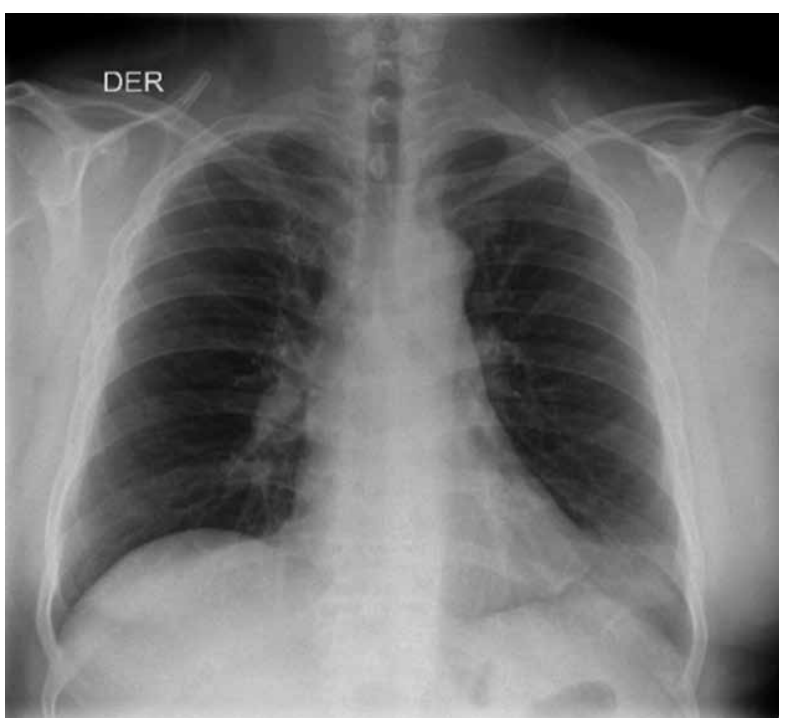

Figura 1: Radiografía de tórax ingreso: Opacidad triangular en la língula, pudiendo corresponder a atelectasia. No se observan otras alteraciones en la transparencia pleuropulmonar. Ángulos costofrénicos libres, no hay signos derrame pleural.
Tabla 2. Resultados de Fibrobroncoscopía.

\begin{tabular}{cc|}
\hline Células epiteliales & 0-2 X campo \\
\hline Leucocitos & $>250 \mathrm{cel} / \mathrm{mm}^{3}$ \\
Neutrofilos & $65 \%$ \\
Linfocitos & $25 \%$ \\
Gram & Negativo \\
Muestra KOH & Negativo \\
\hline Adenosin deaminasa & 6.36 \\
Galactomanan & Neg (negativo) \\
Carga viral CMV & Negativo \\
\hline PCR mycobacterias & Negativo \\
\hline Cultivo para mycobacterias & Negativo \\
\hline Cultivo de gérmenes comunes & \\
\hline
\end{tabular}

con sospecha de etiología fúngica, con baja probabilidad de aspergillosis y se inicia manejo con TMP/SMX $20 \mathrm{mg} / \mathrm{kg} /$ día en sospecha de nocardiosis por hallazgos pulmonares y deterioro neurológico. A las 48 horas posterior de iniciar manejo previo realiza deterioro clínico con traslado a unidad de cuidados intensivos, requerimiento de soporte vasopresor y encefalopatía WH grado III. En los estudios de LBA, el estudio de bloque celular aísla gérmenes en tinciones compatible con pneumocystis

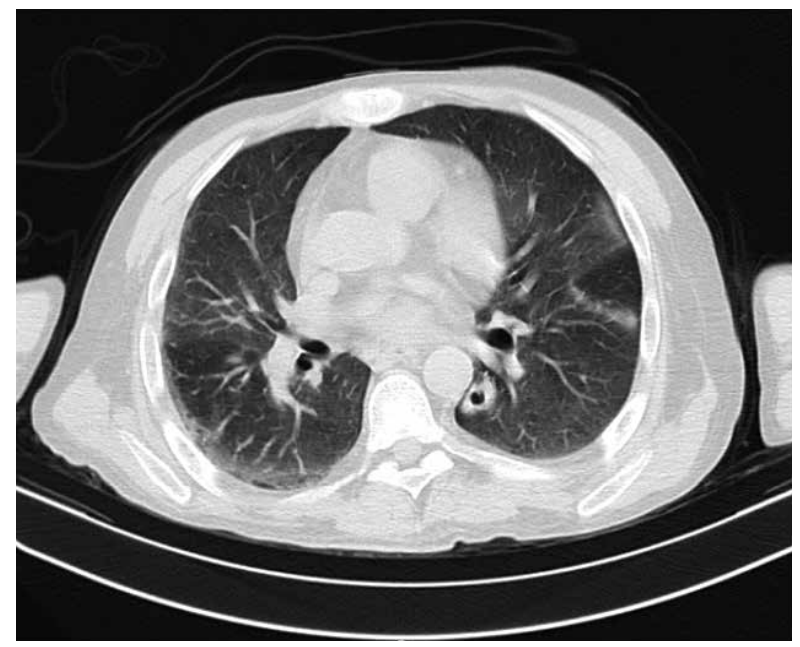

Figura 2: Tomografía axial computarizada de alta resolución que evidencia opacidades en vidrio esmerilado en lóbulos superior e inferior izquierdo y lóbulo inferior derecho de predominio periférico y evidencia de un nódulo cavitado en el segmento superior del lóbulo inferior izquierdo. 


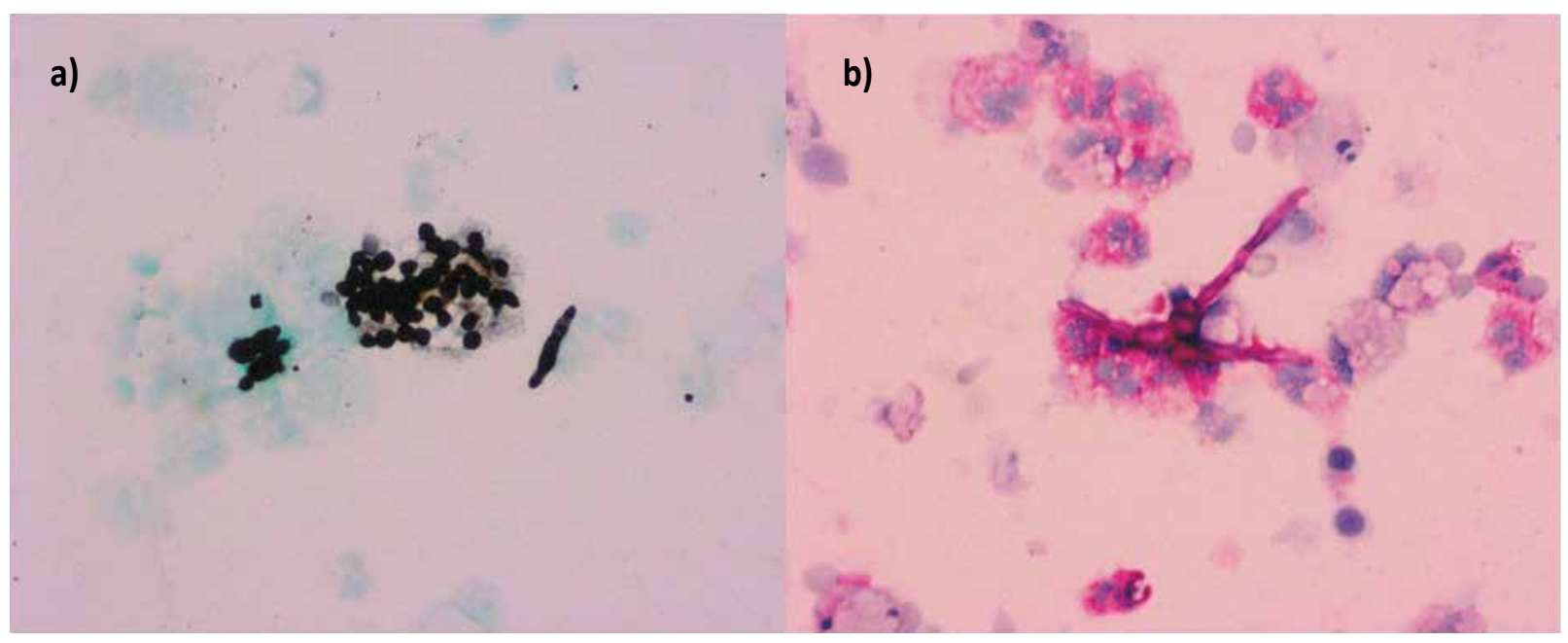

Figura 3: a) Estructuras redondeadas arracimadas de Pneumocystis jirovecii e hifa septada. Coloración de Grocott. b) Hifa septada y ramificada en ángulo de $60^{\circ}$ de Aspergillus fumigatus. Tinción de PAS (Periodic Acid-Schiff).

jirovecci y aspergillus fumigatus (Figura 3). Se da continuidad a manejo con Anfotericina B, TMP/SMX, vancomicina dosis de 30 $\mathrm{mg} / \mathrm{kg}$ dosis de carga continuar $15 \mathrm{mg} / \mathrm{kg}$ cada 12 horas e inicio de esteroide dosis de prednisolona $40 \mathrm{mg}$ cada 12 horas.

En su día 3 de hospitalización realiza deterioro neurológico con focalización dado por paresia del hemicuerpo derecho, por lo cual es llevado a tomografía de cráneo con lesiones focales difusas con distribución compatibles con embolismo séptico no susceptible de manejo quirúrgico; presenta posterior deterioro clínico con trastorno severo de oxigenación, requerimiento de soporte ventilatorio invasivo, aumento de soporte vasopresor con persistencia de pancitopenia y coagulopatia, en contexto de falla hepática aguda sobre crónica Clif-SOFA de 17 puntos-ACLF grado 3, con posterior fallecimiento del paciente en su día 4 de hospitalización. Se revisa posterior hemocultivos negativos, urocultivo negativo, cultivos de LCR - líquido peritoneal negativo, cultivo de LBA negativo (Ver Tabla 1).

\section{DISCUSIÓN}

Pneumocystis jirovecii fue reconocido inicialmente como un patógeno relevante desde la segunda guerra mundial y fue desde 1980 donde toma importancia en su búsqueda activa con la epidemia del VIH. Sin embargo, a pesar de haberse descrito con frecuencia en inmunosuprimidos por SIDA, la infección por Pneumocystis jirovecii suele comprometer pacientes inmunosuprimidos, independiente de su causa. Es así como desde las primeras descripciones ha surgido un incremento en la incidencia y prevalencia de la infección ${ }^{[7]}$ con reportes entre $5 \%-15 \%$ de incidencia en pacientes receptores de trasplante de órgano sólido que actualmente ha disminuido al $1 \%$; y casi el $9 \%$ de pacientes en manejo para neoplasias según un estudio publicado en el $2009^{[8,9]}$. Sin embargo, la evolución de la enfermedad ha llevado también a un incremento en el conocimiento alrededor de la misma, lo cual ha favorecido el entendimiento de los tratamientos profilácticos en pacientes con riesgo de desarrollarla. Esto mismo ha favorecido también el desarrollo de tratamiento óptimo para los pacientes con neumonía por Pneumocystis jirovecii, disminuyendo la mortalidad desde casi el $100 \%$ en pacientes no tratados, hasta máximo un $40 \%$ en pacientes quienes reciben un manejo dirigido ${ }^{[9]}$.

El Pneumocystis jirovecii es un germen unicelular, con componente bifásico en su ciclo de vida (Trofozoito en su fase asexual-proliferativa y quiste en su fase sexual reproductiva), en la actualidad se ha identificado polimorfismos en nucleótidos específicos dando resistencia antibiótico y fallo a profilaxis, se ha identificado principalmente alteraciones en: SOD (Super-óxido dismutasa), CYB ( Citocromo B) y mtLSU rRNA ${ }^{[1,9]}$. Dentro de su fisiopatología se ha identificado desde infección primaria, colonización hasta reactivación de infección latente ${ }^{[10]}$. De una u otra forma, la infección inicia a partir de la adhesión del microorganismo al epitelio de la superficie alveolar en los neumocitos tipo 1 , generando una respuesta inmune que es capaz de eliminar al microorganismo en pacientes inmunocompetentes. Sin embargo, en estado de inmunocompromiso, donde existe un desbalance entre los subtipos de linfocitos T, la ausencia de LT CD4+ causa una respuesta de predominio citotóxica por LT CD8+, desencadenando así un daño epitelial secundario a un estado inflamatorio excesivo ${ }^{[10]}$.

Dentro de sus factores de riesgo se encuentra paciente con $\mathrm{VIH}$, pero en pacientes no VIH se ha identificado la infección por CMV previa, altas dosis de esteroides, trasplante de células hematopoyéticas, trasplante de órgano sólido, enfermedades de tejido conectivo, neoplasias hematológicas, uso de inmunosupresores sintéticos o biológicos ${ }^{[9,11]}$. Se ha descrito la mortalidad en el servicio de hospitalización en paciente con VIH en contexto de neumonía por Pneumocystis jirovecii desde 7 hasta el $26 \%$, pero en pacientes no VIH desde el $29 \%$ hasta 
el $60 \%{ }^{[9]}$. Dentro de las opciones para identificación se cuenta con métodos de aislamiento en LBA con visualización directa en tinciones giemsa, Diff-Quik, Wright en su forma quística o gomori plata metenamina, gram weigert, azul toluidina, blanco calcolfuor en su forma Trofozoito, entre otras tinciones; Adicionalmente se cuenta con galactomanano mayor rendimiento en LBA, y otras técnicas de tinciones con inmunofluorescencia, PCR (reacción cadena polimerasa), amplificación isotérmica mediada por bucle, citometría de flujo, serología IGG - IGM pero en muchas ocasiones se logra el aislamiento por hallazgos en muestras de patología ${ }^{[9]}$. Dentro de sus opciones de tratamiento son en general el blanco de síntesis de ácido fólico, como primera línea el TMP/SMX en el bloqueo de dihidrofolato reductasa y dihidropteroato sintasa. Llama la atención del presente caso el no uso de inmunosupresión previa, con única patología condicionante de inmunosupresión dado por cirrosis previa, en quien el curso fue rápidamente progresivo con desenlace fatal, siendo el galactomanano en LBA negativo y el diagnóstico dado por visualización del germen en bloque celular de líquido de LBA. Específicamente hablando de la co-existencia entre Pneumocystis jirovecii y Aspergillus (siendo más frecuente Aspergillus fumigatus), sabemos que el problema radica en que: 1) En pacientes sin las causas tradicionalmente conocidas de inmunocompromiso $(\mathrm{VIH}$, neoplasias, uso de corticoesteroides o trasplante), la sospecha diagnóstica es baja por la tan poca incidencia y prevalencia y 2) la ausencia de diagnóstico y búsqueda activa de estos microorganismos en dichos pacientes causa una baja tasa de tratamiento óptimo, generando una pobre respuesta terapéutica, lo cual impacta de forma negativa el pronóstico de los pacientes. Todo lo anterior resalta la importancia de este reporte de caso.

Teniendo en cuenta lo anterior, es lógico pensar que no existen en la literatura guías de aproximación y abordaje de pacientes con ambas infecciones de forma simultánea. Los datos existentes se basan en pacientes inmunosuprimidos, y las recomendaciones actuales sugieren la búsqueda del microorganismo que genere mayor sospecha diagnóstica según el comportamiento clínico y radiológico del paciente, y de ser infructuoso el tratamiento, indagar acerca de otras opciones diagnósticas ${ }^{[11]}$.

Sin embargo, es probable que existan métodos por medio de los cuales se puedan detectar ambas infecciones de forma concomitante, especialmente por medio del galactomanano, 0 del B-D-glucano, el primero realizado en el lavado broncoalveolar con un alto rendimiento diagnóstico para infección fúngica invasiva, y el segundo con una muestra en sangre, con mejor rendimiento para neumonía por Pneumocystis jirovecii ${ }^{[12]}$. No obstante, aun con los mejores rendimientos diagnósticos, existe la posibilidad de que resulte negativo el galactomanano en LBA, como en nuestro caso. Entre las principales causas de falsos negativos se encuentran el tratamiento fúngico previo, el cual disminuye la sensibilidad de un $80 \%$ al $20 \%{ }^{[13]}$. Asimismo, al parecer existen también diferencias en la tasa de positividad entre las diversas especies de Aspergillus, al parecer con las tasas más altas resultando en infecciones por $A$. terreus, $A$. niger and $A$. nidulans, un hallazgo que aún debe ser confirmado ${ }^{[13]}$. El tratamiento, una vez sea diagnosticadas ambas infecciones, es realizar tratamiento independiente para cada una de ellas, en lugar de intentar manejo concomitante.

Resumiendo todo lo anteriormente mencionado, y aun con las mejores estrategias diagnósticas, la coinfección de Pneumocystis jirovecii con aspergilosis invasiva representa un pobre pronóstico para el paciente, con una baja probabilidad de sobrevida. El desenlace puede verse afectado con una alta sospecha diagnóstica que guíe los estudios pertinentes para realizar la confirmación de ambas infecciones de forma oportuna.

\section{CONCLUSIÓN}

Presentamos el caso de un paciente sin las causas tradicionales de inmunocompromiso como $\mathrm{VIH}$, trasplante, malignidad o uso de corticoesteroides en quien se diagnostica neumonía infecciosa y se documenta coinfección por Pneumocystis jirovecii concomitante con Aspergillus fumigatus.

Contribución de los autores: CMMM, MAP, y MCFL concibieron la idea de investigación, realizaron el análisis e interpretación de los datos y realizaron la revisión crítica del artículo. Todos los autores participaron en la recolección de los datos y redacción del artículo. Todos los autores participaron en la aprobación de la versión final del manuscrito.

Potenciales conflictos de interés: Los autores declaran no tener conflictos de intereses.

Fuente de financiamiento: Autofinanciado

\section{ORCID:}

Carlos Mauricio Martínez Montalvo, https://orcid.org/00000003-3136-4395

Manuel Antonio Perez, https://orcid.org/0000-0002-2402-6091

María Cynthia Fuentes-Lacouture, https://orcid.org/0000-00024560-4487

Karime Bravo Mena, https://orcid.org/0000-0002-0746-3038

Sofía Fernanda Leal Bernal, https://orcid.org/0000-0002-5208-1960

Yulieth Magdalena Velasco, https://orcid.org/0000-0003-3890-4518

Melissa Charria Caicedo, https://orcid.org/0000-0002-9380-0431

Natalia Estrada Serrano, https://orcid.org/0000-0001-8193-4857

\section{REFERENCIAS BIBLIOGRÁFICAS}

1. Sokulska M, Kicia M, Wesołowska M, Hendrich AB. Pneumocystis jirovecii-from a commensal to pathogen: clinical and diagnostic review. Parasitol Res. 2015;114(10):3577-85.

2. Salzer HJF, Schäfer G, Hoenigl M, Günther G, Hoffmann C, Kalsdorf $\mathrm{B}$, et al. Clinical, Diagnostic, and Treatment Disparities between HIVInfected and Non-HIV-Infected Immunocompromised Patients with Pneumocystis jirovecii Pneumonia. Respiration. 2018;96(1):52-65. 
3. Mariñosa $M$, Soler A, Nogués $X$, Pedro-Botet J. Pulmonary coinfection by Pneumocystis carinii and Aspergillus fumigatus in a seronegative arthritis patient treated with low-dose methotrexate [1]. Clin Rheumatol. 2004;23(6):555-6.

4. Torres HA, Chemaly RF, Storey R et al. Influence of type of cancer and hematopoietic stem cell transplantation on clinical presentation of Pneumocystis jiroveci pneumonia in cancer patients. Eur J Clin Microbiol Infect Dis. 2006;25:382-388.

5. Harris K, Maroun R, Chalhoub M, Elsayegh D. Unusual Presentation of Pneumocystis Pneumonia in an Immunocompetent Patient Diagnosed by Open Lung Biopsy. Hear Lung Circ [Internet]. 2012;21(4):221-4. Available from: http://dx.doi.org/10.1016/j. hlc.2011.10.006

6. Pathak V, Hurtado Rendon IS, Ciubotaru RL. Invasive pulmonary aspergillosis in an immunocompetent patient. Respir Med CME [Internet]. 2011;4(3):105-6. Available from: http://dx.doi. org/10.1016/j.rmedc.2010.12.004

7. Pneumocystis pneumonia | Fungal Diseases | CDC [Internet]. [cited 2021 Sep 21]. Available from: https://www.cdc.gov/fungal/ diseases/pneumocystis-pneumonia/index.html

8. Azoulay É, Bergeron A, Chevret S, Bele N, Schlemmer B, Menotti J. Polymerase chain reaction for diagnosing pneumocystis pneumonia in non-HIV immunocompromised patients with pulmonary infiltrates. Chest. 2009;135(3):655-61.

9. Bateman M, Oladele R, Kolls JK. Diagnosing Pneumocystis jirovecii pneumonia: A review of current methods and novel approaches. Med Mycol. 2020;58(8):1015-28.

10. Castro JG, Morrison-Bryant M. Management of pneumocystis jirovecii pneumonia in HIV infected patients: Current options, challenges and future directions. HIV/AIDS - Res Palliat Care. 2010;2:123-34.

11. Markantonatou AM, loakimidou A, Arvaniti K, Manou E, Papadopoulos V, Kiriklidou $\mathrm{P}$, et al. Pulmonary co-infections by Pneumocystis jirovecii and Aspergillus fumigatus in non-HIV patients: A report of two cases and literature review. Mycoses. 2017;60(10):626-33.

12. Onishi A, Sugiyama D, Kogata $Y$, Saegusa J, Sugimoto T, Kawano $S$, et al. Diagnostic accuracy of serum 1,3- $\beta$-D-glucan for Pneumocystis jiroveci pneumonia, invasive candidiasis, and invasive aspergillosis: Systematic review and meta-analysis. J Clin Microbiol. 2012;50(1):7-15.

13. Verweij PE, Mennink-Kersten MASH. Issues with galactomannan testing. Med Mycol. 2006;44(SUPPL. 1):179-83. 